\title{
Delayed recurrence of hepatocellular carcinoma after liver transplantation: case series
}

\author{
Ta-Hsiang Wong ${ }^{1}$, Cheng-Maw Ho${ }^{2}$, Chih-Yang Hsiao², Yao-Ming Wu², Ming-Chih Ho², Po-Huang Lee ${ }^{2}$, Rey-Heng Hu${ }^{2}$ \\ ${ }^{1}$ Department of School of Medicine, College of Medicine, National Taiwan University, Taiwan \\ ${ }^{2}$ Department of Surgery, National Taiwan University Hospital, Taiwan
}

Background: Hepatocellular carcinoma (HCC) is the third leading cause of cancer deaths worldwide and liver transplant is the definite curative treatment option. However, $15 \%-20 \%$ of recipients will experience HCC recurrence even within stringent Milan criteria, mostly occurring within 2 years. Delayed HCC recurrence after post-transplant 3 years is not commonly observed. We aimed to report the clinical characteristics, risk factor profiles, and post-recurrence survival in patients with HCC recurrence more than 3 years after transplant.

Methods: Patients with HCC recurrence more than 3 years after transplant from February 1999 to December, 2020 in a tertiary university hospital were retrospectively chart-reviewed for underlying medical history, HCC status, treatment, and courses.

Results: Thirty-four out of 195 patients who received liver transplant for HCC had post-transplant HCC recurrence. Five cases occurred more than 3 years after liver transplant. The longest interval was nearly 18 years. For these five late recurrence cases, liver transplant surgeries were all performed in their 50s. The nontumor part of the liver was universally diagnosed with cirrhosis and viral hepatitis (three hepatitis B virus and two hepatitis C virus). Model for end-stage liver disease (MELD) scores were varied between 10-20. Three patients received local treatment (hepatectomy and ethanol injection) for HCC before transplant. Macroscopic or microscopic vascular invasion in explants at the time of liver transplant was noted in two patients. The site of recurrences included liver (3), bone (3), and lung (2); with extra-hepatic recurrence (2), intra-hepatic recurrence (1), and extra- and intra-hepatic recurrence (2). Three patients died in 1-2 years after recurrence. The other two patients are alive, but the follow-up periods are both less than 1 year after recurrence.

Conclusions: One-seventh of post-transplant HCC recurrence occurred more than 3 years after transplant in our series. Though the sample size is too small to reach a definite conclusion, the short post-recurrence survival warrants further clinical investigation.

Corresponding author: Ta-Hsiang Wong

E-mail: b05401091@ntu.edu.tw

(c) The Korean Society for Transplantation

This is an Open Access article distributed under the terms of the Creative Commons Attribution Non-Commercial License (http://creativecommons.org/licenses/by-nc/4.0/) which permits unrestricted non-commercial use, distribution, and reproduction in any medium, provided the original work is properly cited. 